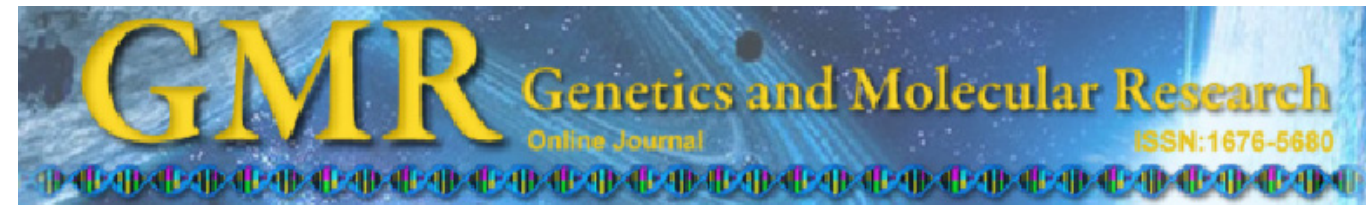

\title{
Role of mitochondrial DNA variants and copy number in diabetic atherogenesis
}

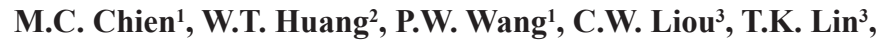 \\ C.J. Hsieh ${ }^{1}$ and S.W. Weng ${ }^{1}$ \\ ${ }^{1}$ Mitochondrial Research Unit, Department of Internal Medicine, \\ Kaohsiung Chang Gung Memorial Hospital, \\ Chang Gung University College of Medicine, Kaohsiung, Taiwan \\ ${ }^{2}$ Department of Pathology, Kaohsiung Chang Gung Memorial Hospital, \\ Chang Gung University College of Medicine, Kaohsiung, Taiwan \\ ${ }^{3}$ Mitochondrial Research Unit, Department of Neurology, \\ Kaohsiung Chang Gung Memorial Hospital, \\ Chang Gung University College of Medicine, Kaohsiung, Taiwan \\ Corresponding author: S.W. Weng \\ E-mail: wenwen@adm.cgmh.org.tw
}

Genet. Mol. Res. 11 (3): 3339-3348 (2012)

Received December 6, 2011

Accepted June 30, 2012

Published September 17, 2012

DOI http://dx.doi.org/10.4238/2012.September.17.4

\begin{abstract}
Hyperglycemia-induced reactive oxygen species production can cause diabetes and its complications, including atherosclerosis. The role of mitochondrial DNA variants and mitochondrial copy number in the pathogenesis of diabetic atherogenesis is not well understood. We examined 36 diabetic patients who had undergone amputation for diabetic foot and seven non-diabetic patients who had undergone amputation after traumatic injury. Mitochondrial DNA was extracted and used for sequencing. Single nucleotide polymorphisms (SNPs) relative to the Cambridge reference sequence were analyzed. Mitochondrial DNA copy number was quantified by real-time PCR. Twenty-one novel variants were detected in 29 diabetic patients with arterial stenosis; six of the variants were heteroplasmic, and most occurred in highly evolutionarily conserved residues. These variants were more prevalent in patients with arterial stenosis than in those without stenosis. The novel
\end{abstract}


variants included four in complex I (ND1: C3477A/C, A3523A/G; ND5: C13028A/C, C13060A/C), one in complex IV (COX1: T6090A/T), and one in rRNA (12srRNA: G857G/T). Compared with non-diabetic patients, the diabetic patients had significantly less mitochondrial DNA. Furthermore, among diabetic patients with arterial stenosis, there was a significant positive correlation between mitochondrial DNA copy number and the number of total SNPs. In conclusion, we identified six novel heteroplasmic mitochondrial DNA variants among diabetic patients with arterial stenosis, and we found that diabetic atherogenesis is associated with decreased amounts of mitochondrial DNA.

Key words: Mitochondrial DNA; Type 2 diabetes mellitus; Atherosclerosis

\section{INTRODUCTION}

Mitochondria are essential organelles that primarily function to support aerobic respiration and to provide energy substrates. Each human cell contains hundreds of mitochondria, each with 2 to 10 copies of mitochondrial DNA (mtDNA), which is small, circular double-stranded DNA of 16,569 bp. mtDNA contains 37 genes, which encode 2 ribosomal RNAs (rRNAs), 22 transfer RNAs (tRNAs), and 13 polypeptides. All of the encoded polypeptides are components of the respiratory chain/oxidative phosphorylation system (Anderson et al., 1981).

Mitochondrial genetics is unique in many ways. First, mtDNA is more susceptible to oxidative damage and has a higher mutation rate than nuclear DNA due to a lack of protective histones, lack of an efficient DNA repair system, and its close proximity to reactive oxygen species (ROS) in mitochondria (Croteau and Bohr, 1997). Specifically, mtDNA is physically associated with the inner mitochondrial membrane, where highly mutagenic oxygen radicals are generated as by-products of oxidative phosphorylation (Allen and Raven, 1996). Second, cells contain hundreds or thousands of mitochondria; since mtDNA is present in multiple copies, pathologic mutations or variants in mtDNA often result in heteroplasmy, the coexistence of both wild-type and mutated mtDNA. Virtually every human organ system can be affected, and many of the pathogenic mtDNA mutations are heteroplasmic. However, most mtDNA SNPs are homoplasmic and are often related to age, neurodegeneration, or metabolic disorders. Some SNPs in control regions are also associated with mitochondrial content (Brownlee, 2001; Wong, 2007; Lu et al., 2010; Tanaka et al., 2010).

Hyperglycemia-induced ROS overproduction is thought to contribute to the onset and progression of diabetes and its complications (Brownlee, 2001; Rosen et al., 2001). A growing body of evidence suggests that oxidative stress plays a key role in the pathogenesis of atherosclerotic cardiovascular disease (Ross, 1993; Puddu et al., 2005; Gutierrez et al., 2006; Madamanchi and Runge, 2007). Hyperglycemia-induced formation of advanced glycation end-products can generate ROS (Chappey et al., 1997); ROS are associated with somatic DNA mutations, which may contribute to atherogenesis (Rosen et al., 2001; Brownlee, 2001). Several genetic polymorphisms may also play a role in the development and progression of atherosclerosis in diabetic patients. The mitochondrial respiratory chain is the major source of ROS production, contributing to progressive mitochondrial dysfunction and mitochondrial 
damage clinically associated with atherosclerosis. Moreover, hyperglycemia-induced overproduction of ROS by endothelial mitochondria has been implicated in glucose-mediated vascular damage (Nishikawa et al., 2000; Du et al., 2003).

Although mitochondria are a major source of ROS and can be compromised by oxidative stress, few studies have investigated the association between mitochondrial mutations and the pathogenesis of atherosclerosis. Therefore, to clarify their roles in diabetic atherogenesis, we examined mtDNA variants and mtDNA content in the atherosclerotic vessels of patients with diabetic foot.

\section{MATERIAL AND METHODS}

\section{Patients}

We recruited 36 patients with type 2 diabetes mellitus (DM) and 7 non-diabetic controls. All patients with type $2 \mathrm{DM}$ had received leg amputations due to unhealed leg ulcers. The control patients had received leg amputations due to traumatic injuries.

On the basis of pathologic examination, we classified atherosclerosis into 4 groups depending on the level of arterial occlusion: grade 0 was $0 \%$; grade 1 was $0-25 \%$; grade 2 was $25-50 \%$, and grade 3 was more than $50 \%$. The prevalence of arterial stenosis from grade 0 to grade 3 in the diabetic group was 19.4 (7/36), $13.9(5 / 36), 27.8(10 / 36)$, and 38.9\% (14/36), respectively. In contrast, there was no arterial stenosis in the non-diabetic group. There were 29 patients with arterial stenosis and 14 without stenosis. We searched for novel sequence variants and compared mtDNA copy number in each group.

The studies were conducted according to the guidelines of the Declaration of Helsinki, and the protocol for this study was approved by the Ethics Committee of the Chang Gung Memorial Hospital. All subjects provided written informed consent.

\section{Laboratory methods}

\section{Mitochondrial genome analysis}

mtDNA was extracted from blood vessels embedded in paraffin blocks and was used for sequencing to screen for sequence variants.

DNA for sequence analysis of the entire mitochondrial genome was amplified as 32 overlapping segments of approximately $500-1100$ bp by a symmetric PCR method. The sequence primer was a 38-mer oligonucleotide, consisting of an 18-bp sequence of a universal forward sequencing primer (-21M13, 5'-TGTAAACGCGGCCAGT-3') and a 20-bp L-strandspecific sequence at the 5 '-end. PCR amplifications were carried out in a final reaction volume of $20 \mu \mathrm{L}$ containing $200 \mathrm{ng}$ of the first PCR product, $10 \mathrm{mM}$ Tris- $\mathrm{HCl}, \mathrm{pH} 8.3,50 \mathrm{mM} \mathrm{KCl}$, $1.5 \mathrm{mM} \mathrm{MgCl}, 0.2 \mathrm{mM}$ of each dNTP, $1.0 \mu \mathrm{M}$ of each primer, and $0.5 \mathrm{U} p f u$ DNA polymerase (Takara Bio Inc.). The PCR conditions were as follows: initial denaturation at $95^{\circ} \mathrm{C}$ for $5 \mathrm{~min}$, 40 cycles of denaturation at $95^{\circ} \mathrm{C}$ for $35 \mathrm{~s}$, annealing at $60^{\circ} \mathrm{C}$ for $40 \mathrm{~s}$, and extension at $72^{\circ} \mathrm{C}$ for $1 \mathrm{~min}$, and a final extension at $72^{\circ} \mathrm{C}$ for $10 \mathrm{~min}$. The PCR fragments were analyzed by $2 \%$ agarose gel electrophoresis and were visualized by staining with ethidium bromide. The PCR products were sequenced with an ABI Big Dye Terminator (version 3.1) cycle sequencing 
ready reaction kit and an $\mathrm{ABI} 3730 \mathrm{XL}$ sequencer (Applied Biosystems, Foster City, CA, USA) according to manufacturer instructions. Novel variants and SNPs in the mitochondrial genome relative to the Cambridge reference sequence were analyzed by the DNASTAR and Bio Edit Sequencing Analysis Software.

\section{Quantification of mtDNA copy number by real-time PCR}

The relative mtDNA copy numbers were measured by real-time PCR and normalized by simultaneous quantification of nuclear DNA. PCR was performed in an ABI PRISM 7700 sequence detection system (PE Biosystems, CA, USA) using a SYBR green PCR master mix kit (Applied Biosystems, NJ, USA). The forward and reverse primers for $\beta$-actin, the nuclear gene, were 5'-TCACCCACACTGTGCCCATCTAGGA-3' and 5'-CAGCGGAACCGCTCAT TGCCAATGG-3'. The forward and reverse primers for ND1, an mtDNA gene sequence, were: 5'-TGGGTACAATGAGGAGTAGG-3' and 5'-GGAGTAATCCAGGTCGGT-3'. PCR was performed for 40 cycles with $10 \mathrm{ng}$ DNA in a $12.5-\mu \mathrm{L}$ reaction mixture using an SYBR green PCR master mix kit and $50 \mathrm{nmol}$ forward and reverse primers. PCR cycling conditions were a 15 -s denaturation step at $95^{\circ} \mathrm{C}$, a 20 -s annealing step at $60^{\circ} \mathrm{C}$, and a 15 -s extension step at $72^{\circ} \mathrm{C}$. A melting curve was performed for $20 \mathrm{~min}$ after the real-time PCR, and analysis was performed using the Dissociation Curve Software. The amplified products were denatured and reannealed at different temperatures to detect their specific melting temperatures. The threshold cycle number $(\mathrm{Ct})$ values were determined in the same quantitative PCR run. The results were confirmed by a second run. $\mathrm{Ct}$ values were used as a measure of the copy number, and $\mathrm{Ct}$ value differences were used to quantify mtDNA copy number relative to $\beta$-actin, calculated as follows: relative copy number $(\mathrm{Rc})=2^{\Delta \mathrm{Ct}}$, where $\Delta \mathrm{Ct}=\mathrm{Ct}_{\beta \text {-actin }}-\mathrm{Ct}_{\mathrm{ND} 1}$. There was good agreement in $\mathrm{Ct}$ values within and between runs.

\section{Statistical analysis}

Data were logarithmically transformed, because the relative mtDNA copy number values were not normally distributed. We used $t$-tests and analysis of variance for further comparison and analysis. Data are reported as means $\pm \mathrm{SD}$. Relationships between different variables were analyzed by the Pearson correlation test. $\mathrm{P}<0.05$ was considered to be statistically significant.

\section{RESULTS}

\section{Novel mtDNA variants}

A total of 28 novel variants in srRNA, tRNA, and coding regions were detected when compared to the Mitomap database. These variants included 3 in srRNA and 2 in tRNA genes. Another 23 variants were within coding regions; of these, 8 were non-synonymous, and 15 were synonymous. Of all the variants, 7 were heteroplasmic.

Among the 28 novel variants, 21 were identified in diabetic patients with arterial stenosis; 4 were identified in diabetic patients without arterial stenosis, and 4 were identified in non-diabetic patients. One variant, A3140G, was identified in both diabetic patients with arterial stenosis and non-diabetic patients. There was no difference in the prevalence of the novel 
variants between the diabetic and non-diabetic groups or between those with or without arterial stenosis. However, patients with arterial stenosis harbored more heteroplasmic mutations $(7 / 29=24.1 \%)$ than those without arterial stenosis $(1 / 14=7 \%)$.

\section{$m t D N A$ variants in diabetic patients with arterial stenosis}

Table 1 summarizes the novel mtDNA variants in diabetic patients with arterial stenosis and Figure 1 illustrates the highly conserved novel mtDNA variants in diabetic patients.

\begin{tabular}{|c|c|c|c|c|}
\hline \multirow[t]{2}{*}{ Patient code } & \multicolumn{4}{|c|}{ Diabetic patients with arterial stenosis } \\
\hline & Novel variant & Gene affected & ho/he & Amino acid change \\
\hline \multicolumn{5}{|l|}{ Complex I } \\
\hline D30 & $\mathrm{C} 3477 \mathrm{~A} / \mathrm{C}$ & ND1 & he & $\mathrm{Thr} \rightarrow \mathrm{Thr}$ \\
\hline D31 & A3523A/G & ND1 & he & $\mathrm{Thr} \rightarrow$ Ala \\
\hline D5 & C3930A & ND1 & ho & $\mathrm{Val} \rightarrow \mathrm{Val}$ \\
\hline D33 & A10523G & ND4L & ho & Gly $\rightarrow$ Gly \\
\hline D13 & $\mathrm{T} 10652 \mathrm{C}$ & ND4L & ho & $\mathrm{Ile} \rightarrow \mathrm{Ile}$ \\
\hline D24 & $\mathrm{C} 10870 \mathrm{~T}$ & ND4 & ho & $\mathrm{Ile} \rightarrow \mathrm{Ile}$ \\
\hline D18 & C11602T & ND4 & ho & Asp $\rightarrow$ Asp \\
\hline D25 & $\mathrm{C} 13028 \mathrm{~A} / \mathrm{C}$ & ND5 & he & Pro $\rightarrow$ His \\
\hline D25 & $\mathrm{C} 13060 \mathrm{~A} / \mathrm{C}$ & ND5 & he & Pro $\rightarrow$ Thr \\
\hline D30 & C14130T & ND5 & ho & $\mathrm{Thr} \rightarrow \mathrm{Thr}$ \\
\hline \multicolumn{5}{|l|}{ Complex IV } \\
\hline D3 & $\mathrm{A} 6035 \mathrm{~T}$ & COX1 & ho & Pro $\rightarrow$ Pro \\
\hline D13 & $\mathrm{T} 6090 \mathrm{~A} / \mathrm{T}$ & $\operatorname{COX} 1$ & he & $\mathrm{Phe} \rightarrow \mathrm{Ile}$ \\
\hline D15 & $\mathrm{T} 6090 \mathrm{~A} / \mathrm{T}$ & COX1 & he & $\mathrm{Phe} \rightarrow \mathrm{Ile}$ \\
\hline D13 & C6797T & COX1 & ho & Asp $\rightarrow$ Asp \\
\hline D22 & C7223T & COX1 & ho & $\mathrm{Tyr} \rightarrow \mathrm{Tyr}$ \\
\hline D25 & C9929T & $\mathrm{COX} 3$ & ho & $\mathrm{Tyr} \rightarrow \mathrm{Tyr}$ \\
\hline \multicolumn{5}{|l|}{ Complex V } \\
\hline D4 & A8985G & ATPase6 & ho & Pro $\rightarrow$ Pro \\
\hline D31 & A8536G & APTase6/ATPase8 & ho & $\mathrm{Asn} \rightarrow \mathrm{Asp} / \mathrm{Glu} \rightarrow \mathrm{Glu}$ \\
\hline \multicolumn{5}{|l|}{ rRNA } \\
\hline D30 & $\mathrm{G} 857 \mathrm{G} / \mathrm{T}$ & 12srRNA & & \\
\hline D26 & A3140G & 16srRNA & & \\
\hline D5 & 3168InsC & 16srRNA & & \\
\hline D10 & A7542G & tRNA (Aspartate) & & \\
\hline D10 & T15943C & tRNA (Threonine) & & \\
\hline
\end{tabular}

ho $=$ homoplasmic variant; he $=$ heteroplasmic variant.

Among the 3 novel variants in NADH dehydrogenase subunit 1 (ND1) of complex I, 2 $(\mathrm{C} 3477 \mathrm{~A} / \mathrm{C}$ and $\mathrm{A} 3523 \mathrm{~A} / \mathrm{G})$ were heteroplasmic. The $\mathrm{A} 3523 \mathrm{~A} / \mathrm{G}$ variant caused a threonineto-alanine amino acid substitution. In NADH dehydrogenase subunit 5 (ND5), 2 heteroplasmic variants $(\mathrm{C} 13028 \mathrm{~A} / \mathrm{C}$ and $\mathrm{C} 13060 \mathrm{~A} / \mathrm{C})$ caused proline-to-histidine and proline-to-threonine amino acid substitutions, respectively. The $\mathrm{C} 13028 \mathrm{~A} / \mathrm{C}$ and $\mathrm{C} 13060 \mathrm{~A} / \mathrm{C}$ variants may be detrimental, because they occur at highly conserved amino acid residues, which have been conserved from amphibians to mammals.

There were 5 novel variants in complex IV; the T6090A/T variant was heteroplasmic and resulted in a phenylalanine-to-isoleucine amino acid substitution. This variant may be detrimental, because it also occurred at a highly conserved amino acid residue.

In complex $\mathrm{V}$, the $\mathrm{A} 8536 \mathrm{G}$ variant was homoplasmic and was located in a region with overlapping coding sequences for ATPase6 and ATPase8. As a result of this variant, there was an 


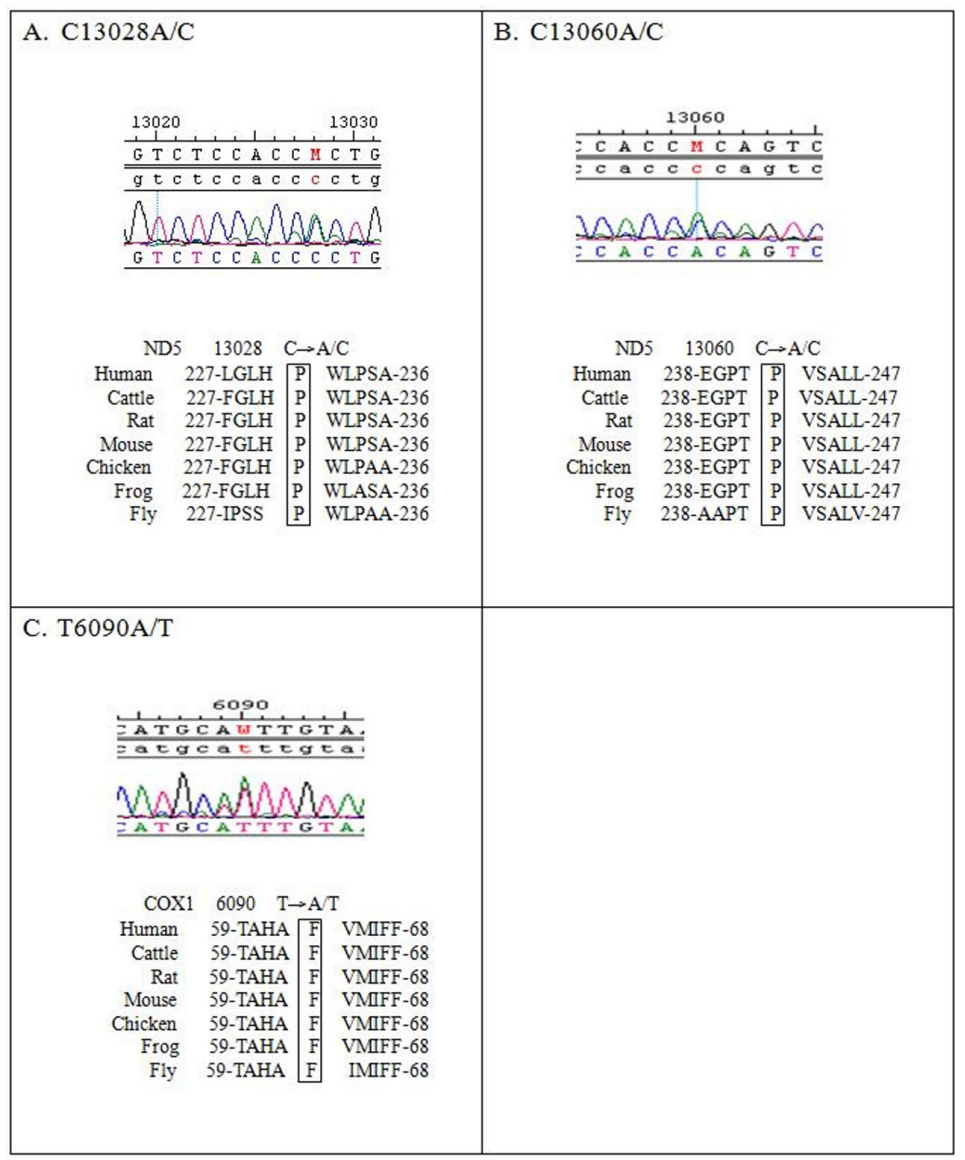

Figure 1. Highly conserved novel mtDNA variants were detected in diabetic patients using the direct sequencing method described in the Materials and Methods section. A. The C13028A/C variant was detected in patient D25. The variant caused a proline-to-histidine amino acid substitution in NADH dehydrogenase subunit 5 (ND5). B. The $\mathrm{C} 13060 \mathrm{~A} / \mathrm{C}$ variant was also found in patient D25. The variant in ND5 resulted in a proline-to-threonine amino acid substitution. C. Diabetic patients D13 and D15 harbored the T6090A/T variant of complex IV.

asparagine-to-aspartic acid amino acid substitution in ATPase6. This variant may also be detrimental, as it occurred at an amino acid residue that is evolutionarily conserved among mammals.

The G857G/T, A3140G, and 3168InsC variants were detected in rRNA genes, and the homoplasmic A7542G and T15943C variants were found in tRNA genes.

In conclusion, among 29 diabetic patients with arterial stenosis, a total of 21 novel variants were found. The variants causing amino acid substitutions often occurred at highly conserved residues.

\section{mtDNA variants in patients without arterial stenosis}

For diabetic patients without arterial stenosis, all novel variants (C3585T, A4844G, 
A4902G, and T15066A) were homoplasmic, but some - A4902G and T15066A variants caused isoleucine-to-valine and phenylalanine-to-tyrosine amino acid substitutions. However, these substitutions did not occur at highly conserved amino acid residues.

Among the 7 non-diabetic patients, 4 had mtDNA variants: C3528T, T15183A/T, C6395T, and A3140G in complex I, III, and IV, and rRNA genes, respectively. The T15183A/T variant was heteroplasmic, whereas the others were homoplasmic. The T15183A/T variant occurred at a highly conserved amino acid residue and resulted in an isoleucine-to-asparagine amino acid substitution.

Therefore, among the 14 patients without arterial stenosis, there were a total of 8 novel variants, most of which were homoplasmic.

\section{Group differences in mtDNA content and SNP number}

Diabetic patients had significantly fewer copies of mtDNA than did non-diabetic patients $(2.55 \pm 0.15$ vs $2.73 \pm 0.16, \mathrm{P}=0.008)$. Patients with arterial stenosis (grades 1,2 , and 3) also had significantly fewer copies of mtDNA compared to those without arterial stenosis (grade $0 ; 2.54 \pm 0.13$ vs $2.66 \pm 0.20, \mathrm{P}=0.035$ ). This difference was most pronounced in patients with severe arterial stenosis (grade 3; $2.53 \pm 0.08 v s 2.66 \pm 0.20, \mathrm{P}=0.043$; Figure 2). Furthermore, there was a positive correlation between the total number of SNPs and mtDNA copy number $(\mathrm{r}=0.433, \mathrm{P}=0.019)$ in diabetic patients with arterial stenosis. There was a similar positive correlation between mtDNA copy number and the number of control region SNPs $(r=0.362, \mathrm{P}=0.053$; Figure 3$)$.
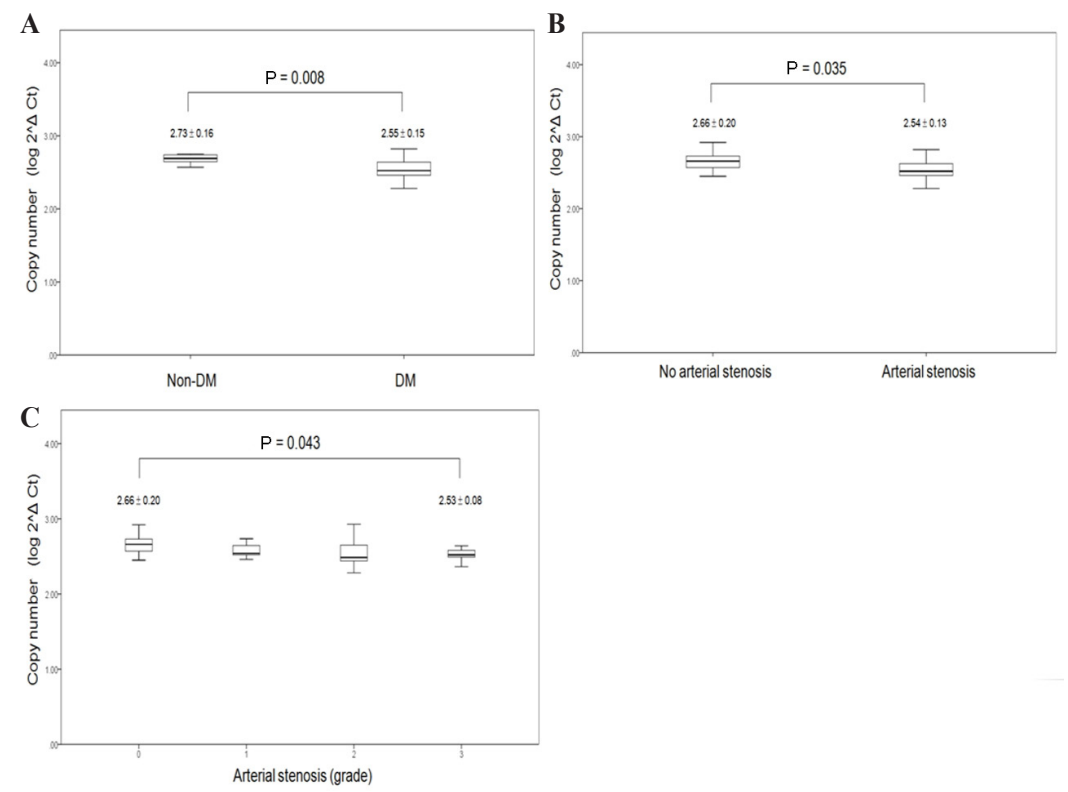

Figure 2. Comparison of mtDNA content in diabetic (DM) patients, non-diabetic (non-DM) patients, and patients with arterial stenosis. A. Diabetic patients had fewer copies of mtDNA than non-diabetic patients $(\mathrm{P}=0.008)$. B. Patients with arterial stenosis had a lower mtDNA content than those without arterial stenosis $(P=0.035)$. . Patients with the most severe arterial stenosis had a significantly lower mtDNA content than those without arterial stenosis $(\mathrm{P}=0.043)$. 
A
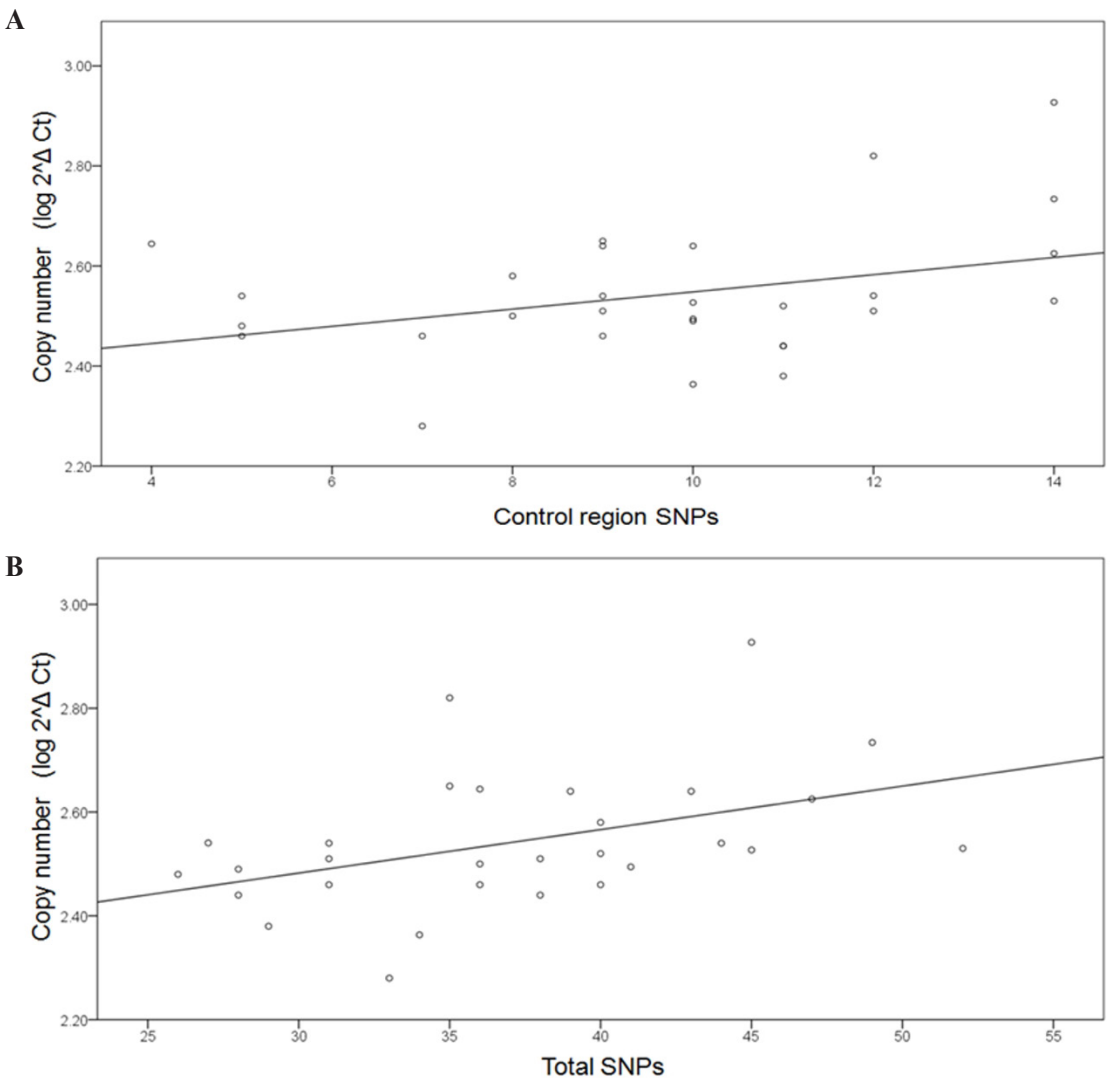

Figure 3. Relationship between single nucleotide polymorphisms (SNPs) and mitochondrial content in diabetic patients with arterial stenosis. A. There was a positive correlation $(\mathrm{r}=0.362, \mathrm{P}=0.053)$ between the number of control region SNPs and mtDNA content in diabetic patients with arterial stenosis. B. mtDNA content was significantly correlated with the total number of SNPs in the diabetic group $(r=0.433, P=0.019)$.

\section{DISCUSSION}

In animals, mtDNA damage, increased ROS, and reduced oxidative phosphorylation may result in the failure of DNA repair and lead to changes in glucose and lipid metabolism, thus promoting atherosclerosis (Mercer et al., 2010).

In this study, we found that there were more heteroplasmic variants in patients with arterial stenosis, most of which occurred in evolutionarily conserved regions and resulted in amino acid substitutions. Moreover, more tRNA and rRNA variants were found in patients with arterial stenosis compared to those without arterial stenosis. Only one such variant was found in the non-stenotic group.

Among the novel mtDNA variants possibly affecting oxidative phosphorylation, the C13028A/C (ND5), C13060A/C (ND5), T6090A/T (COX1), A8536G (ATPase6/ATPase8), and $\mathrm{T} 15183 \mathrm{~A} / \mathrm{T}$ (Cytb) variants caused amino acid substitutions in highly conserved regions. The T6090A/T variant is likely to play a role in atherogenesis, because it was found in 2 unre- 
lated diabetic patients with arterial stenosis. Although the A8536G variant was homoplasmic, it was located in a region of overlap between the ATPase 6 and ATPase8 genes and resulted in an amino acid substitution in ATPase6. Among the novel rRNA variants, the G857G/T variant is potentially detrimental. First, it was heteroplasmic in the diabetic patients but not in the nondiabetic patients. Second, A856G, a variant near G857G/T, has been clinically associated with mitochondrial disorders (Sawano et al., 1996; Tanaka et al., 2010; Lu et al., 2010). Thus, the $\mathrm{G} 857 \mathrm{G} / \mathrm{T}$ variant is potentially pathogenic. There was another variant in the $16 \mathrm{~s}$ rRNA gene, 3168InsC; however, its impact on protein synthesis is presently unknown.

In the present study, one diabetic patient with atherosclerosis had both an A7542G transition in the aspartate tRNA mitochondrial gene and a T15943C transition in the threonine tRNA mitochondrial gene. The presence of multiple polymorphisms in tRNA genes has not been previously reported. Importantly, the A7542G mitochondrial variant was located in the anticodon hairpin region of the aspartate tRNA gene; an adjacent tRNA variant, A7543G, was previously associated with myoclonic epilepsy and psychomotor regression (Shtilbans et al., 1999). The T15943C transition in the threonine tRNA gene was located in the T arm loop region, where several neighboring polymorphisms have been previously identified. Moreover, an adjacent variant, T15940del, was identified in a family with mitochondrial myopathy (Seneca et al., 1998; Tuppen et al., 2008). We hypothesize that A7542G and T1594C are potentially detrimental variants.

Our present findings revealed a lower mtDNA copy number in diabetic patients, particularly in patients with severe atherosclerosis. The mechanisms of disease progression and complications in diabetic patients are probably multi-factorial; one factor may be hyperglycemia-induced overproduction of ROS by mitochondria. Increased oxidative stress is known to cause mitochondrial damage, dysregulation of mitochondrial function, and altered mitochondrial biogenesis (Lee et al., 2000, 2002). Petersen et al. (2004) showed that insulin-resistant offspring of parents with type $2 \mathrm{DM}$ had impaired mitochondrial function associated with severe muscle insulin resistance. In a follow-up study, they proposed that reduced muscle mitochondrial content contributed to the decreased mitochondrial activity in insulin-resistant subjects. Patients with type $2 \mathrm{DM}$ have decreased copies of mtDNA in insulin-resistant target tissues, such as skeletal muscle and adipose tissue (Morino et al., 2005). In the present study, we demonstrated an association between decreased mtDNA copy number and diabetic atherogenesis.

Among diabetic patients with arterial stenosis, who had a lower level of mtDNA, the numbers of control region SNPs and total SNPs were positively correlated with increased mitochondrial copy number. Impaired mitochondrial function may stimulate mitochondrial biogenesis, as a possible feedback response compensating for defective mitochondria in the face of respiratory chain impairment. We hypothesize that an increase in total SNPs may be a compensation for lower mtDNA content in atherosclerotic vessels of diabetic patients, implying that a change in SNPs may play a role in overcoming stress. However, we were unable to determine the specific SNPs or haplotypes related to diabetic atherosclerosis due to our relatively small sample size.

In conclusion, our study identified several novel potentially harmful mtDNA variants in diabetic atherogenesis. We also demonstrated that diabetic atherogenesis is associated with decreased mtDNA content. Furthermore, an increase in total SNPs may compensate for reduced mitochondrial content. 


\section{ACKNOWLEDGMENTS}

Research supported by the National Science Council, Executive Yuan (ROC; \#NSC98-2314-B-182A-017-MY2). The authors acknowledge the technical services (support) provided by the Sequencing Core Facility of the National Yang-Ming University Genome Research Center (YMGC). The Sequencing Core Facility is supported by the National Research Program for Genomic Medicine (NRPGM), National Science Council.

\section{REFERENCES}

Allen JF and Raven JA (1996). Free-radical-induced mutation vs redox regulation: costs and benefits of genes in organelles. J. Mol. Evol. 42: 482-492.

Anderson S, Bankier AT, Barrell BG, de Bruijn MH, et al. (1981). Sequence and organization of the human mitochondrial genome. Nature 290: 457-465.

Brownlee M (2001). Biochemistry and molecular cell biology of diabetic complications. Nature 414: 813-820.

Chappey O, Dosquet C, Wautier MP and Wautier JL (1997). Advanced glycation end products, oxidant stress and vascular lesions. Eur. J. Clin. Invest. 27: 97-108.

Croteau DL and Bohr VA (1997). Repair of oxidative damage to nuclear and mitochondrial DNA in mammalian cells. $J$. Biol. Chem. 272: 25409-25412.

$\mathrm{Du}$ X, Matsumura T, Edelstein D, Rossetti L, et al. (2003). Inhibition of GAPDH activity by poly(ADP-ribose) polymerase activates three major pathways of hyperglycemic damage in endothelial cells. J. Clin. Invest. 112: 1049-1057.

Gutierrez J, Ballinger SW, Darley-Usmar VM and Landar A (2006). Free radicals, mitochondria, and oxidized lipids: the emerging role in signal transduction in vascular cells. Circ. Res. 99: 924-932.

Lee HC, Yin PH, Lu CY, Chi CW, et al. (2000). Increase of mitochondria and mitochondrial DNA in response to oxidative stress in human cells. Biochem. J. 348: 425-432.

Lee HC, Yin PH, Chi CW and Wei YH (2002). Increase in mitochondrial mass in human fibroblasts under oxidative stress and during replicative cell senescence. J. Biomed. Sci. 9: 517-526.

Lu J, Li Z, Zhu Y, Yang A, et al. (2010). Mitochondrial 12S rRNA variants in 1642 Han Chinese pediatric subjects with aminoglycoside-induced and nonsyndromic hearing loss. Mitochondrion 10: 380-390.

Madamanchi NR and Runge MS (2007). Mitochondrial dysfunction in atherosclerosis. Circ. Res. 100: 460-473.

Mercer JR, Cheng KK, Figg N, Gorenne I, et al. (2010). DNA damage links mitochondrial dysfunction to atherosclerosis and the metabolic syndrome. Circ. Res. 107: 1021-1031.

Morino K, Petersen KF, Dufour S, Befroy D, et al. (2005). Reduced mitochondrial density and increased IRS-1 serine phosphorylation in muscle of insulin-resistant offspring of type 2 diabetic parents. J. Clin. Invest. 115: 3587-3593.

Nishikawa T, Edelstein D, Du XL, Yamagishi S, et al. (2000). Normalizing mitochondrial superoxide production blocks three pathways of hyperglycaemic damage. Nature 404: 787-790.

Petersen KF, Dufour S, Befroy D, Garcia R, et al. (2004). Impaired mitochondrial activity in the insulin-resistant offspring of patients with type 2 diabetes. N. Engl. J. Med. 350: 664-671.

Puddu P, Puddu GM, Galletti L, Cravero E, et al. (2005). Mitochondrial dysfunction as an initiating event in atherogenesis: a plausible hypothesis. Cardiology 103: 137-141.

Rosen P, Nawroth PP, King G, Moller W, et al. (2001). The role of oxidative stress in the onset and progression of diabetes and its complications: a summary of a Congress Series sponsored by UNESCO-MCBN, the American Diabetes Association and the German Diabetes Society. Diabetes Metab. Res. Rev. 17: 189-212.

Ross R (1993). The pathogenesis of atherosclerosis: a perspective for the 1990s. Nature 362: 801-809.

Sawano T, Tanaka M, Ohno K, Yoneda M, et al. (1996). Mitochondrial DNA mutations associated with the 11778 mutation in Leber's disease. Biochem. Mol. Biol. Int. 38: 693-700.

Seneca S, Lissens W, Liebaers I, van den Bergh P, et al. (1998). Pitfalls in the diagnosis of mtDNA mutations. J. Med. Genet. 35: 963-964.

Shtilbans A, El-Schahawi M, Malkin E, Shanske S, et al. (1999). A novel mutation in the mitochondrial DNA transfer ribonucleic acidAsp gene in a child with myoclonic epilepsy and psychomotor regression. J. Child Neurol. 14: 610-613.

Tanaka N, Goto Y, Akanuma J, Kato M, et al. (2010). Mitochondrial DNA variants in a Japanese population of patients with Alzheimer's disease. Mitochondrion 10: 32-37.

Tuppen HA, Fattori F, Carrozzo R, Zeviani M, et al. (2008). Further pitfalls in the diagnosis of mtDNA mutations: homoplasmic mt-tRNA mutations. J. Med. Genet. 45: 55-61.

Wong LJ (2007). Pathogenic mitochondrial DNA mutations in protein-coding genes. Muscle Nerve 36: 279-293. 